\title{
Energy Efficient Group Messaging for Mobile Instant Messenger Apps
}

\author{
Pinial Khan Butt ${ }^{1}$, Wang Yang ${ }^{1 *}$, Md Zakirul Alam Bhuiyan ${ }^{2}$ and Guojun Wang ${ }^{3,1}$ \\ ${ }^{1}$ School of Information Science and Engineering, Central South University, \\ Changsha, China, 410083 \\ ${ }^{2}$ Department of Computer and Information Science, Temple University, \\ Philadelphia, PA 19121 \\ ${ }^{3}$ School of Computer Science and Educational Software, Guangzhou University, \\ Guangzhou, 510006, China \\ pinial@yahoo.com,yangwang@mail.csu.edu.cn, zakirulalam@gmail.com, \\ csgjwang@mail.csu.edu.cn
}

\begin{abstract}
Recent technology advancement in smartphones makes it the most important device in daily life communication. Instant messenger (IM) applications (apps) such as WhatsApp, WeChat, Viber and $Q Q$ messenger are gaining users demand for communication. We take data collected from WeChat and $Q Q$ which are most popular IM application as an example to investigate their traffic pattern. Based on our experimental findings, group chat messages take a larger portion in our data collected messages. We find users experiencing shorter battery life after group chat messages are in background traffic. In this paper, we look into improving group chat energy consumption. Our proposed energy efficient group chat messages support network preferences such as $3 G$ or WiFi network. In addition, group chat traffic pattern is dependent on user status awareness, knowledge; we postponed messages over time while user is inactive and deliver postponed messages when user is active in group chats. We evaluate that our energy efficient group chat messaging achieves energy saving between $13.7 \%$ to $32 \%$ for $3 G$ and $21 \%$ to $27 \%$ for WiFi, users.
\end{abstract}

Keywords: Instant messaging, group chat, energy efficient, network traffic, smartphone

\section{Introduction}

A smartphone is a powerful computer that fits in a pocket. The latest smartphone capabilities have increased tremendously. Smartphones allow you to do almost everything desktop, laptop, and computers can do and make calls as well. The latest development in smartphones is changing people's life. Now people can do tasks using smartphone which cannot possibly be done using a desktop, laptop or a computer such as smartphone GPS sensor that can determine locations and plot directions. The increased smartphones sales in 2011 surpassed the desktop computers sales number which shows usage of smartphones as the more prevalent platform [1]. As the usage of smartphone increases, it also boosts up apps available on app store market. Google play digital store has more than 700,000 numbers of apps available for download. Windows Phone app store is catching up with more than 150,000 apps available to users [2]. Unfortunate as the usage of smartphone increases, so do problems about battery life. Smartphone is still powered by limited battery capacity. A normal user of smartphone must charge it many times in a day. So in order to extend battery lifetime users manually need to turn off hardware

${ }^{*}$ Corresponding Author 
components such as GPS, 3G, WiFi, NFC and Bluetooth when they are not in use. This leads to user frustration when having to enable different components on or off in smartphone.

Network interfaces (such as WiFi, 3G and LTE) consumes the most energy in smartphones. Previous studies [3-5], found that the background services such as email when synchronizing continuously uses the network interface to exchange data with the corresponding server; even when there is no input of smartphone user. There is a recent increased usage of apps in smartphone especially daily life activities such as entertainment, games and instant messaging (IM) etc., IM, WeChat, WhatsApp and QQ apps already have 438, 400, 800 million online users [6, 7]. Instant messenger apps constantly use smartphone network interface for real-time text transmission over the Internet. Obviously, disruptive network traffic data transmission through network interface needs to be optimized to save battery power. IM apps aperiodic network traffic pattern data transmission can drain smartphone battery relatively quickly due to latency in $3 \mathrm{G}$ and other network interface overheads.

A technique is proposed by researchers to delay tolerant applications such as email and RSS (Rich Site Summary) feeds where data transmission can be delayed and batched to reduce tail time [8]. However, such an approach is not suitable for more interactive applications such as instant messenger; otherwise users may suffer from delayed messages. In terms of cost, instant messenger is free but in terms of energy cost, it can drain smartphone battery very quickly as instant messenger apps are delivering real-time content like text, images, voice chat and video chat from single chat (one to one) or to a group chat (one to many) in most aperiodic time intervals. Instant messenger (IM) applications (apps) provide functionalities in single chat (one to one) such as online presence or user status awareness (active or inactive user in chat) with typing notification. IM user status awareness (active or inactive user in chat) could affect traffic pattern mode (e.g., the screen is on / off, the smartphone user active in instant messenger chat or inactive switch to focus on another application). But in group chat (one to many) lacking these functionalities and unaware without user status awareness traffic pattern has potential impact on energy consumption.

When a $3 \mathrm{G} / \mathrm{WiFi}$ is off state changes to turn on state, smartphone $3 \mathrm{G} / \mathrm{WiFi}$ generate network traffic. In this point, instant messenger (IM) app traffic can be classified into 4 distinct modes. When smartphone user active in instant messenger chats, it is called foreground traffic. Foreground traffic has two modes, which are sending and receiving. Foreground traffic sending (FTS); When IM user app is running in the foreground, this time period is considered when user type messages to be sent to other IM users. Foreground traffic receiving (FTR); When IM user app is running in the foreground, in this time period IM user receives a message sent by other IM users. When smartphone user is inactive in instant messenger and switch to focusing other application, it is called background traffic. This is further divided into two modes; background traffic with a screen on and background traffic with the screen off. Background traffic with a smartphone screen on (BTSON); When IM app is running in the background; In this time period screen is on, but receive other messages in the background. Background traffic with smartphone screen off (BTSOFF); IM app is running in background, similar to BTSON mode, but here continues smartphone screen is off and it continues to receive messages in background.

In this paper, we focus solely on improving the energy efficiency of instant messenger group chat traffic pattern. Due to wastage of battery power currently practiced by IM app group chat. IM app group chat mainly, QQ and WeChat most users receive many messages in background traffic. Improvement in term of group chat user status awareness (user active or inactive in chat) traffic pattern can play a role in lowering energy consumption in a smartphone.

In summary, in this paper we make the following contributions: 
- We present a most popular IM data collected from WeChat and QQ chat conversations and data collection study of instant messenger. It reveals many IM users receive messages in background traffic from a group chat, which leads to high energy consumption in a smartphone. Our data set differs from previous work [6] in two aspects. Firstly, our experimental evidence target group chat traffic pattern in instant messenger app. Secondly, our data collected is based on the most popular WeChat and QQ instant messenger.

- Proposed energy efficient group chat messages support network preference such as $3 \mathrm{G}$ or WiFi network and traffic pattern is dependent on user status awareness can save energy between $13.7 \%$ to $32 \%$ for $3 \mathrm{G}$ and $21 \%$ to $27 \%$ for $\mathrm{WiFi}$, users.

The focus of research work is about group chat (one to many) traffic pattern. Therefore, we do not consider single chat (one-to-one) text conversations or multimedia messages, which can be an extension for future research work. To our knowledge, we are the first contribution towards achieving energy efficient group chat in instant messenger apps.

The organization of the paper is as follows. Section 2 presents work that has been done in the area of energy efficient in a smartphone. In section 3, we present investigation of 36 IM users data collection. In section 4, energy efficient group chat. In section 5, evaluation to proposed energy efficient group chat, measurement tool, and test environment to get results about proposed traffic pattern energy saving cost. Section 6 concludes the paper and future work.

\section{Related Works}

In recent years, there has been a growing interest in studying energy efficient solution for smartphone battery energy consumption. We discuss the related work in several categories.

\section{- Context-aware approach to reducing power consumption}

A smartphone has many built-in sensors such as accelerometer and barometer etc., Archan Misra and Lipyeow Lim have used algorithms to pull data streamed from the sensors rather than push data. It optimizes $70 \%$ reduce energy overhead of continuous query processing such as from sensor phone [9]. Another study, about human-computer interaction used sensors to get user activity recognition [10]. A prior study [11] addresses the problem to minimize smartphone battery energy consumption by using smartphone sensors to recognize user activity such as user fitness health, context awareness and share user events or moments on a social network. Previous studies [12, 13] improves energy consumption of Global Positioning System (GPS) application on smartphone.

\section{- Power measuring Tool}

Introduces [14] PowerTutor which is a power monitoring application into smartphone hardware components. Another tool, Appscope is used to trace power at kernel level [15]. Previous studies [16-18] used power model approach to measure energy consumption on smartphones. The most famous mobile Nokia company also contributed a power tool called Nokia Energy Profiler. Intel Power Gadget [19] is a software-based power usage monitoring tool enabled for 2 nd Generation Intel Core processors or later. It is supported on Windows and Mac OS X. The Monsoon power tool hardware and software provide a robust power measurement solution for mobile devices [20]. Application Resource Optimizer (ARO) [21], a free diagnostic tool analyzing cross-layer information to detect radio resource and energy inefficiencies in Radio Resource Control (RRC) state machine. 


\section{- Pre-fetching traffic and delay-tolerance techniques}

Designed method [14] pauses data transmission in smartphone operating system level and gather enough burst network traffic. A proposal [22] proactive traffic based on predict human behavior to offloading traffic through WiFi network. It is acceptable to prefect traffic to reduce network active mode time and keep network interface in an idle status longer.

\section{- Multiple interfaces to reduce energy consumption}

Several studies have been carried out to investigate multiple network interfaces in smartphones. Prior studies [23-27] use multi network interface such as Bluetooth, WiFi and $3 \mathrm{G} / \mathrm{LTE}$ to increase the through output and illustrate how to reduce energy consumption. Designed [28] system called Wiffler which switches to 3G if WiFi access point reception is poor quality in moving a vehicle. It transfers more data on WiFi but switches to $3 \mathrm{G}$ if there is sensitive data transfer in case of delay or loss. To understand this existing study, they performed an experiment to cost user equipment power usage $[29,30]$. They found $3 \mathrm{G}$ and WiFi are more energy efficient than LTE for a small amount of data transfer. The result, for transferring one packet power usage for LTE, $3 \mathrm{G}$ and $\mathrm{WiFi}$ is $12.76 \mathrm{~J}, 7.38 \mathrm{~J}$ and $0.04 \mathrm{~J}$ respectively. This shows LTE is not energy efficient solution for small amount of data transfer. As a result, WiFi is more energy efficient solution for small amount of transfer and $3 \mathrm{G}$ fall behind it. LTE is energy efficient for bulk data transfer. The recent $3 \mathrm{G}$ users increased by 38.251 million and reached 166.675 million [31]. To reduce an amount of data transfers, can optimize energy in joules used to transfer these packets. We delay unnecessary data transfer in $3 \mathrm{G}$ connecting environment and later download it on WiFi. It helps optimize power usage of a smartphone app to processing network usage. A retrieving data in cellular mobile network consumes more power if not cached.

\section{- The present study in instant messenger}

Regular SMS (Short Message Service) by carriers also has been suppressed due to IM (instant messenger) app which provides more functionalities such as real-time text and multimedia exchange and other user status awareness. Prior studies [32-33] investigates instant messenger data collected to extract evidence crime scene from chat messages. For the past few years, a few research focus on energy prospect in instant messenger. A previous study reveals 5 famous instant messenger bandwidth utilization inefficiencies in conversion and background receiving messages [34]. A previous study [35] investigated data collected from IM 51 users single chat (one to one) text messages. IM end user typed multiple messages and bundled together to user end typing to reduce the energy cost of transmission packets. This is the only research that has been previously done to investigate energy consumption in the context of instant messenger single chat (one to one) chatting.

The study research on IM group chat (one to many) features for energy cost has been neglected. IM group chat messages traffic pattern inefficiencies cannot be neglected. A group chat is mostly denser and small peaks packet transmission over smartphone network interfaces can drain the battery more quickly.

\section{Data Collection}

Tencent is the most popular services technologies used for online gaming, e-commerce, according and social networks. We selected to investigate WeChat and QQ which are IM (instant messenger) apps used for text messages or multimedia messages. The focus of this work is on text messages, therefore, we do not consider multimedia messages. We are interested to analyzing traffic generated by instant messenger without user interaction, which translate into smartphone network interface energy consumption. This can reveal 
IM traffic pattern inefficiencies in term of energy consumption in a smartphone. IM data collected consists of real 36 users single chat (one to one) and group chat (one to many) text messages conversations that were collected between January 14, 2015 to December 14, 2015. The collected IM text messages conversion was encrypted by 256-bit Advanced Encrypted Standard key. A parser was used for parsing messages from individual user smartphone. IM data collected logs period contained 16 QQ and 20 WeChat instant messenger accounts and individual user device conversions. QQ is the most popular instant messenger used in China. The latest QQ international version has a million downloads and has gained international users attraction due to its great feature of language translation. Regarding ages of QQ IM user data collected: users were between 18 to 24 years, 6 users were between 25 to 30 and 2 users were between 31 to 40 . Regarding QQ IM users country, 14 users were from China and 2 users from Pakistan. WeChat IM app was launched by Tencent in 2011, but it has potential gain in user growth. According to 2015 statics, it has 600 million monthly active users [34]. Regarding ages of WeChat IM users data collected: 12 users were between 18 to 24 years, 4 users were between 25 to 30 and 4 users were between 31 to 40. Regarding WeChat IM users country, 10 users were from China, 4 users from Pakistan and 2 from Russians, 2 from England and 2 from Kenya. The purpose to investigate 36 IM data collected sent / received messages was to reveal traffic pattern instant messenger inefficiencies in term of energy consumption. IM collected diverse users chat data collected contains messages sent / received direction, type of messages (single or group chat ), a number of group chats subscribed, message distribution per day per group chat, group chat $3 \mathrm{G}$ cellular and WiFi network data usage, Group chat traffic pattern characteristic and inefficiencies in group chat. We are focusing on group chat traffic pattern inefficiencies in term of energy consumption.

\subsection{Classification of Messages}

3.1.1. QQ Instant Messenger: In Figure 1, shows 16 QQ IM data collected per user single and group chat sent / received messages. The most users single chat received messages portion is larger than single chat sent messages. Except user 7 which have single chat received $39 \%$ and single chat sent $61 \%$ messages. All users have single chat received $59 \%$ compare to single chat $41 \%$ sent messages. Regarding the single chat received standard deviation is $4.9 \%$ and single chat sent standard deviation is $3.35 \%$. According to a number of users, messages chat standard deviation user 4 has lowest single chat received messages and highest single chat received messages are user $8,9,13,14,15$ and 16 . User 12 has the lowest single sent messages and user $1,2,3,5,6,7,9,11$ have the highest single chat sent messages.

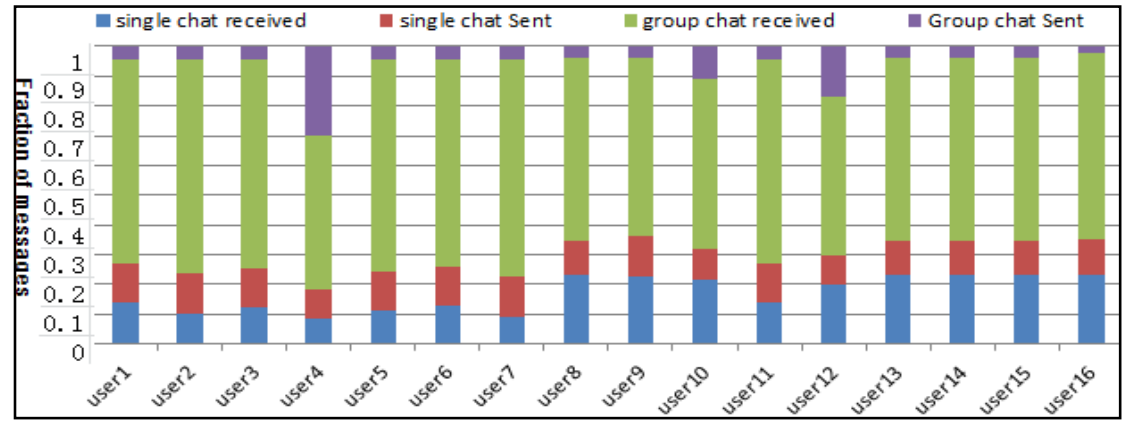

Figure 1. Classification of $Q Q$ Instant Messenger Chat Messages 


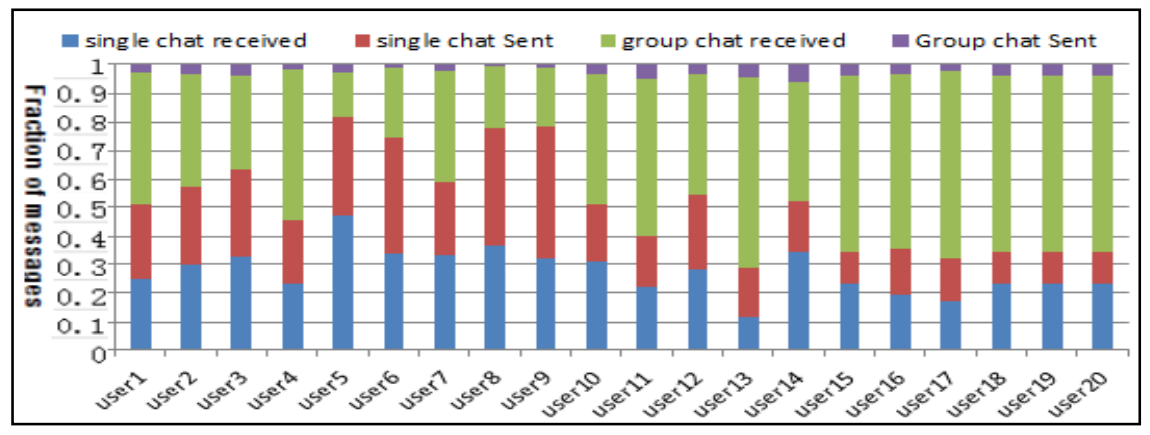

Figure 2. Classification of WeChat Instant Messenger Chat Messages

Looking into group chat traffic messages, all of the users have a much larger proportion of group chat received messages compared to group chat sent messages. For example, user 8 total traffic has $1 \%$ group chat sent messages and $99 \%$ group chat received messages. Except for user 4, which has group chat received at $41 \%$ and group chat sent at 59\%. All users group chat received has 92\% compare to group chat sent $8 \%$ sent messages. Regarding the group chat received standard deviation is $3.39 \%$. According to standard deviation, group chat received messages; user 4 has the lowest received messages and users 1,2,3,4,5,6,7 has the highest group chat received messages. In comparison among group chat sent messages, user 16 has lowest and user 4 has the highest group chat sent messages.

3.1.2. WeChat Instant Messenger: In Figure 2, shows WeChat IM data collected per user messages classification. WeChat IM has total 20 users single chat received $51 \%$ and $49 \%$ single chat sent messages. Further group chat traffic messages all of the users has a much larger proportion of group chat received messages compared to group chat sent messages. For example, users from 1 to 20 total traffic has $94 \%$ group chat received messages and $6 \%$ group chat sent messages. The standard deviation for all users group chat received messages are $2 \%$ and $1.7 \%$. The users 5,8,9 has lowest group chat received messages and user 13 has highest group chat received messages. The user 8 has lowest group chat sent messages and 3,11,14 has highest group chat sent messages.

\subsection{IM Group Chat Characteristics}

The use of IM apps has been widely adopted as a group conversation. The increased use of IM group chat has been a source to understand messages traffic pattern impact on battery energy consumption. We exclude further sent messages analysis in group chat. In previous section 3.1 we find group chat has few sent messages compare to many receive messages has a high potential impact on battery energy consumption. The instant messenger apps such as WeChat and QQ messenger have a single chat (one to one) chatting and (one to many) users chatting called group chat. Further, to initiate own user group chat (one to many) conversations. IM users can create group chat simply by selecting multiple users from their IM contact list and dragging them into a group chat. After formal acceptance to participate in group chat. Moreover, any of the users in group chat conversion can also invite their user contacts list into the same group chat conversion. Although group chat initiator user has right to delete any one or quit group chat any time; by default, chat messages are turned on to all users in group chat conversion without knowing user status awareness in chat. 


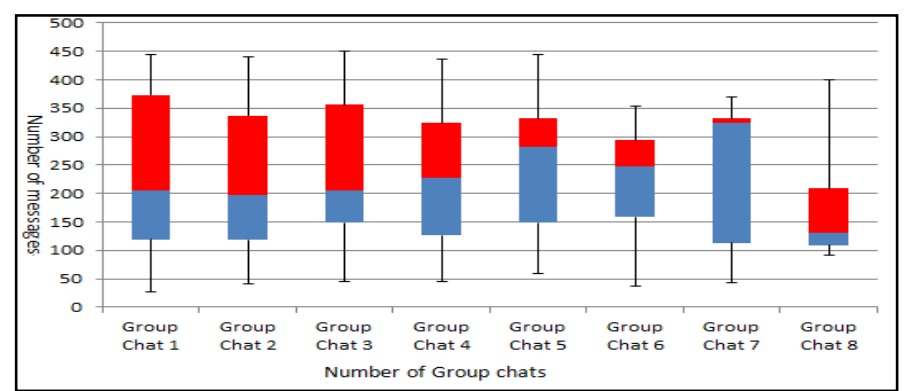

Figure 3. Message Distribution Per Day Per Group Chat

3.2.1. Message Distributions Per Group Chat: In this, we describe receive messages per group chat. In Figure 3 shows a number of messages receive per day per group chat. There is a great diversity in received messages across group chats. From very active group chat, there are more than 400 messages per day and less active group chat have less than 18 messages per day. In group chats 1,2 and 3 average receive messages range between 190 to 210 . The group chats $4,5,6,7$ and 8 has average receive messages varies from each other. The maximum average receive messages are 331 in group chat 7 . The minimum average receive messages are 130 in group chat 8. IM users subscribed to group chats ranges between 1 to 8 groups. One group chat is common among these users. The maximum group chat users size is 500. The minimum group chat users size is 38. Group chat interest are learning English, learning Chinese, artwork, traveling events, classmates chat and jobs opportunity discussion.

3.2.2. Network Data Usage: The group chat data transmission by network preferences such as $3 \mathrm{G}$ and WiFi network can influence energy consumption in a smartphone. A 3G cellular network due to tail effect consumes more energy than WiFi network. Figure 4 we find $36 \mathrm{IM}$ users used up to $75 \%$ percent WiFi received a byte, rather than only $25 \%$ on $3 \mathrm{G}$ cellular received byte data. Moreover, IM data $3 \mathrm{G}$ cellular sent byte was $8 \%$ and WiFi sent byte was $91 \%$. This shows mostly IM app users used WiFi to connect with the internet. In addition, received traffic is much greater than sent traffic in group chat. Furthermore, currently IM user cannot choose network preference $3 \mathrm{G}$ cellular or WiFi network in IM app to send / receive chat messages.

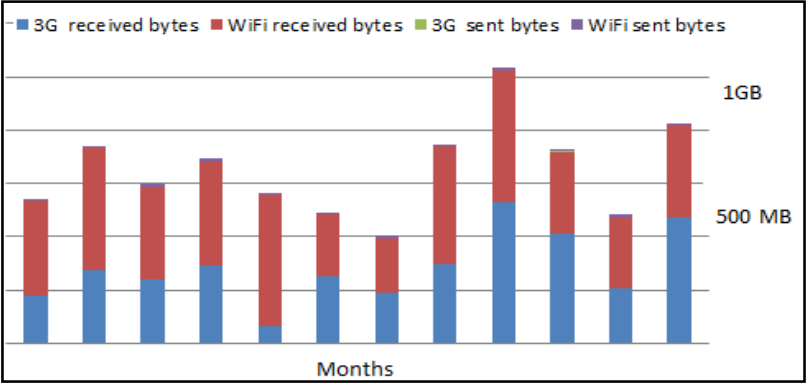

Figure 4. 3G Cellular and Wifi Network Data Usage

3.2.3. Group Chat Traffic Pattern Inefficiencies: In this section, we describe if received messages are in background traffic as this traffic pattern leads to high energy consumption in a smartphone. The group chat user active in instant messenger receives messages causes foreground traffic receiving (FTR) and user inactive in instant messenger causes background traffic receiving screen on / off (BATSON) or (BTSOFF). Group chat background traffic receiving with a screen on or off (BTSON or BTSOFF) due to IM user inactive in application causes battery energy consumption wastage. IM user is chatting in 
group chat. A user might experience after reading messages or sent messages that user is not participating in chatting, but user is continuously receiving in background messages from other IM users chat conversion in group chat. We found in group chat traffic pattern, IM user have received many messages without knowing user status awareness. IM data collected in every chat message distinguish between by its timestamp, we found subsequent group chat messages last seen by IM users left a timestamp in chat. Figure 5, shows the group chat receive messages active user represent foreground traffic receiving (FTR) and inactive user receive messages represent as background traffic with a screen on or off (BTSON or BTSOFF). Further IM active user foreground traffic receiving (FTR) per day 3.5\% and inactive user background traffic screen on or off (BTSON or BTSOFF) per day is $96.5 \%$ of total message in 365 days. We can observe in Figure 5 foreground traffic receiving (FTR) messages is much less than user inactive background traffic screen on or off (BTSON or BTSOFF). Most user inactive during background traffic screen on or off (BTSON or BTSOFF) in group chat. But we cannot distinguish fair enough user inactive in instant messenger background traffic receiving messages when user smartphone screen is (BTSON) on or (BTSOFF) off. IM apps group chat in background traffic receiving screen on or off (BTSON or BTSOFF) depends on other group chat users sent messages. Therefore, received message background traffic screen on or off (BTSON or BTSOFF) IM user inactive in group chat leads to high energy consumption. We found activate turn off notification option in IM app about group chat messages from WeChat and QQ IM. IM app group chat just only do not notify users names on screen and mute messages receiving sound but it keeps busy data transmission via network interface receives messages causes background traffic screen on or off (BTSON or BTSOFF). The group chat receives messages traffic pattern of instant messenger is not determining mainly by user interaction. Most instant messenger users have low bandwidth communication. IM user that inactive time period has irregular data transmission which leads to higher energy consumption due to network overheads. But it is quite complex to make it energy efficient due to delay in real time traffic that can halt user experience. So it cannot be aggregating, batch or of similar kind of techniques.

We observed that group chat messaging interval overall was denser. The energy perspective, one after the other group messages receive traffic wastes a lot of energy of network interface since there is no user interaction. Furthermore, in background receiving these messages lead to higher energy consumption. In IM background traffic receiving one after the other group messages elapsed time is greater than inactivity time; smartphone battery consumes another energy tail effect in $3 \mathrm{G}$. We investigated that user generally does not frequently look into group chat messages. But it receives many messages in a denser manner. In an account of energy perspective, any user inactive in group chat receive messages could inefficiently drain battery power since small burst has high latency in $3 \mathrm{G}$. In our data collected analysis, IM user mostly inactive from group chat the most received traffic run in background traffic with a screen on or off (BTSON or BTSOFF). It aggressively causes smartphone battery drain for IM user inactive in group chat.

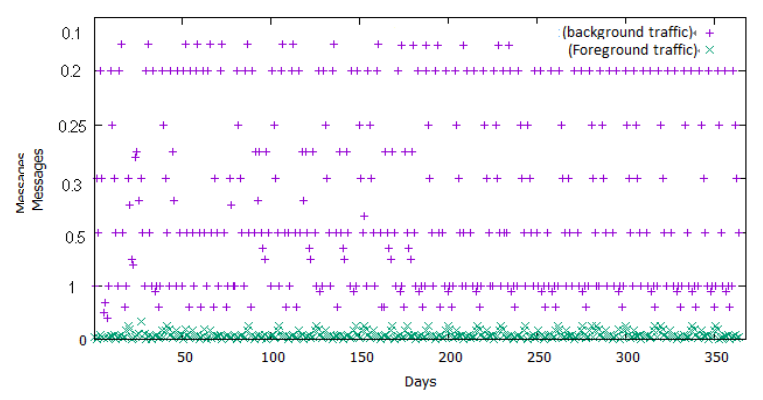

Figure 5. User Active (Foreground Traffic) and User Inactive (Background Traffic) In Group Chat 


\section{Energy Efficient Group Chat}

User status awareness should be integrated with current group chat instant messenger applications. The QQ and WeChat applications data collected analyze shows significantly lacking to support data transmission by network prefer such as $3 \mathrm{G}$ cellular or WiFi network. In addition, the traffic pattern is not dependent on user status awareness in group chat. Our proposed group chat messages support data transmission choose to prefer $3 \mathrm{G}$ cellular or WiFi only network. However, IM user can send messages any time but receive messages postponed when IM user inactive and receive messages deliver when user active in IM group chat. The potential benefit is optimized network data transmission and prevent group chat receive messages running in background traffic screen on or off (BTSON or BTSOFF), this leads to low energy consumption in a smartphone. IM app users group chat messaging choose $3 \mathrm{G}$ or WiFi network preference details in subsection 4.1. Traffic pattern based on IM user status awareness in group chat details in subsection 4.2. Workflow sending and receiving in group chat in subsection 4.3

Figure 6, shows energy efficient group chat. Details description below:

- System message: Means to detect IM users network information (3G cellular or WiFi) and user active or user inactive in group chat.

- Personal message send: Means personal communication messages. User can send messages in group chat.

- Personal message received: Means personal communication messages. User can receive messages in group chat.

- User active: A user active in IM group chat. A time period user is active in group chat. User is able to send and receive messages.

- User inactive: A user inactive in IM group chat. A time period user is inactive in group chat. User does not want to send or receive messages.

- Group Chat server: Group Chat Server consist nth tier distributed server environment to manage user account authentication, profile, database etc., Group Chat server uses system message to ping user status and type of network information ( $3 \mathrm{G}$ cellular or WiFi).

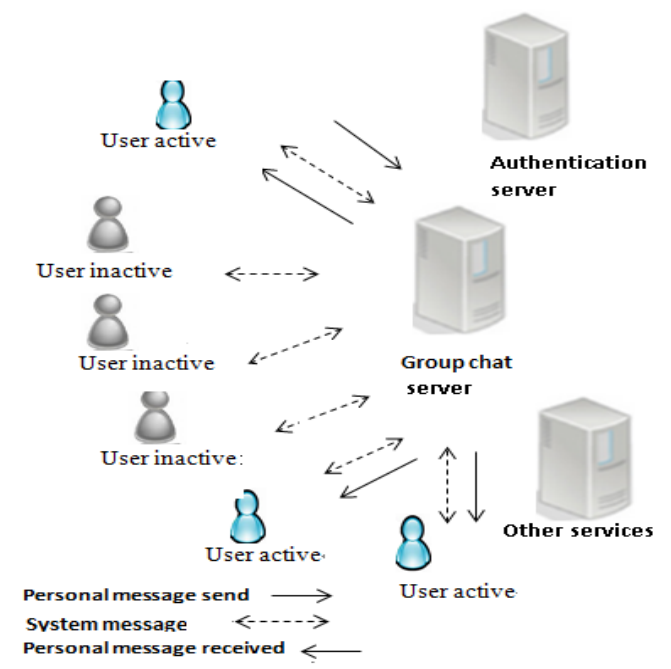

Figure 6. Energy Efficient Group Chat Traffic Pattern Conceptual Model 


\subsection{Network Preferences}

User chooses to prefer $3 \mathrm{G}$ cellular when traveling but prefer WiFi when at home or work. In QQ and WeChat IM app scenario, IM user cannot choose to 3G cellular or WiFi network. But in our proposed energy efficient group chat, IM user can set 3 options for network preference data transmission such as $3 \mathrm{G}$ cellular or WiFi network. In energy perspective, it reduces energy consumption when data transfer in WiFi. IM user may experience important messages postponed. IM user choose to prefer WiFi only and IM user using $3 \mathrm{G}$ cellular then consecutive messages queued at chat server. In our proposal, overcome a negative impact on IM user QoE (Quality of Experience). IM user can be notified at the time to start a group chat. IM user who chooses $3 \mathrm{G}$ cellular or WiFi to start group chat time expires in 24 hours. Chat server asks again after 24 hours by sending a notification to IM user to start group chat if any new messages queued for delivery in subscribed group chat. The network preferences $3 \mathrm{G}$ cellular or WiFi network options 1 to 3 listed below.

User preference1.Start group chatting over 3G cellular or WiFi network availability. (May consume more energy in 3G cellular. Please present in group chat. Otherwise, you will not receive messages).

User preference 2. Start group chat only over WiFi network. (Please present in group chat. Otherwise, you will not receive messages).

User preference 3. Retrieve chat messages history within 7 days.

NOTE: User can ignore this message. A user does not want to participate in group chat.

Network preferences description is in details. The user can ignore this notification to start group chat by chat server. A user does not want to participate in group chat. However, a user can choose among the 3 options. Each group chat is identified by its name or unique identity. IM user choose preference 1 and reply back to the chat server, it starts group chat in $3 \mathrm{G}$ cellular or WiFi network availability. In this case user, group chat messages in $3 \mathrm{G}$ cellular may consume more energy. IM user choose preference 2 , chat server postponed or store in database consecutive message delivery up to system messages predict user smartphone use WiFi. IM user choose preference 3, this prefer use to retrieve chat messages history from chat server within expire 7 days either in a $3 \mathrm{G}$ cellular or WiFi network.

\subsection{User State Awareness Traffic Pattern}

After IM app user choose network preference to start a group chat. Group chat traffic pattern now dependent on user status awareness in group chat, because it can prevent running group chat receive messages in background traffic screen on or off (BTSON or BTSOFF). IM user is inactive in group chat. It is fair enough to postpone consecutive messages until user active again in group chat. In energy perspective, it reduces energy consumption in a smartphone.

Variable definition Assume that we have a group $G_{1}$. Let user status awareness denoted by $\beta \in[1,0]$, where 1 shows user active and 0 shows user inactive in group chat. $M=\left\{m_{1}, \ldots, m_{n}\right\}$ is sequence of messages receive by smartphone. Where $T=\left\{t_{1}, \ldots, t_{n}\right\}$ is total time spend to transmit M messages. $\phi P=\left\{p_{1}, \ldots, p_{n}\right\}$ denote the total energy consumption cost spend on successful transmission of $\mathrm{M}$ messages to smartphone. The problem is to schedule $\mathrm{M}$ transmission to $G_{1}$ on a smartphone to minimize $\phi P$ smartphone energy consumption. We solve the problem as follows. Introducing postponed messages for user inactive in group chat. Benefits are that it 
saves lot of joules. When multiple users send messages to chat, user inactive from group chat messages postponed queued at chat server. When $\beta=0$, total group chat energy consumption should be equal to zero $\phi P=0$ due to no $m_{n}$ messages transfer when IM user inactive in group chat. When $\beta=1$, total energy consumption is denoted as $\phi P=\left\{p_{1}, \ldots, p_{n}\right\}$. Given a traffic pattern sequence $P_{t}=\left\{p_{t 1}, . ., p_{t n}\right\}$, where $P_{t}$ is a traffic pattern set with $n$ patterns, and user status $\beta \in$ between one and zero [1,0]. M group chat user have status $\beta \in[1,0]$ is directly proportional sequence of messages $M=\left\{m_{1}, \ldots, m_{n}\right\}$ data transmission in each traffic pattern occurrences $P_{t}=\left\{p_{t 1}, . ., p_{t n}\right\}$ from chat server to smartphone. A user is active in group chat, when chat server receives messages for delivery. Group chat messages queue for delivery at chat server up to user inactive turn in to active in group chat. In order to analyze the relationship between postponed $M$ message in the traffic pattern and energy cost of group chat $\phi P$, we use the following correlation formula equation 1. Correlation coefficient $r$ satisfies -1 if $r$ value closer to -1 represents a negative correlation between $M$ and $\phi P$.

$$
\rho(M, \phi P)=\frac{\operatorname{Cov}(M, \phi P)}{\sqrt{\operatorname{Var}(M) \operatorname{Var}(\phi P)}}
$$

The sample correlation coefficient $\mathrm{r}$ between two samples $M$ and $\phi P$ is defined as equation 2 .

$$
r=S_{M \phi P} / \sqrt{S_{M M} S_{\phi P \phi P}}
$$

Table 1. Test Conversion Messages

\begin{tabular}{|r|l|l|l|}
\hline Serial no & time & messages & messages contents \\
\hline 1 & $\mathrm{t}_{1}$ & $\mathrm{~m}_{1}$ & Hi \\
\hline 2 & $\mathrm{t}_{2}$ & $\mathrm{~m}_{2}$ & Let go to watch a movie. \\
\hline 3 & $\mathrm{t}_{3}$ & $\mathrm{~m}_{3}$ & Happy new year everyone. \\
\hline 4 & $\mathrm{t}_{4}$ & $\mathrm{~m}_{4}$ & Good luck \\
\hline 5 & $\mathrm{t}_{5}$ & $\mathrm{~m}_{5}$ & bye \\
\hline
\end{tabular}

\subsection{Test Conversion}

Five different test conversions are observed in Table 1 . We use Table 1 test conversion to postponed messages during user inactive time and receive them on user active time to generate different group chat traffic pattern. The results of different group traffic pattern is observed in Table2. Table 2 shows traffic pattern $P_{t}=\left\{p_{t 1}, p_{t 2}, p_{t 3}, p_{t 4}, p_{t 5}\right\}$ are different from each other. Table 2. group chat traffic pattern details given below.

\section{Group chat traffic pattern}

Pattern $p_{t 1}$. User has status $\beta=1$ at time $t_{1}$. So total message generated and received is $M=\left\{m_{1}\right\}$

Pattern $p_{t 2}$. User has status $\beta=0$ at time $t_{1}$. Total postponed message is $M=\left\{m_{1}\right\}$.When user active at time $t_{2}$. Total received messages are $M=\left\{m_{1}+m_{2}\right\}$ 
Table 2. Group Chat Traffic Patterns

\begin{tabular}{|c|c|c|c|c|}
\hline $\begin{array}{c}\text { Pattern } \\
\text { No. }\end{array}$ & $\begin{array}{c}\text { user active } \\
\beta=1\end{array}$ & $\begin{array}{c}\text { user inactive } \\
\beta=0\end{array}$ & $\begin{array}{c}\text { postponed } \\
\text { Messages }\end{array}$ & Messages Size \\
\hline$p t 1$ & $\mathrm{t}_{1}$ & $\mathrm{~N} / \mathrm{A}$ & $\mathrm{m}_{1}$ & 3 byte \\
\hline$p t 2$ & $\mathrm{t}_{2}$ & $\mathrm{t}_{1}$ & $\mathrm{~m}_{1}+\mathrm{m}_{2}$ & 26 byte \\
\hline$p t 3$ & $\mathrm{t}_{3}$ & $\mathrm{t}_{1}, \mathrm{t}_{2}$ & $\mathrm{~m}_{1}+\mathrm{m}_{2}+\mathrm{m}_{3}$ & 52 byte \\
\hline$p t 4$ & $\mathrm{t}_{4}$ & $\mathrm{t}_{1}, \mathrm{t}_{2}, \mathrm{t}_{3}$ & $\mathrm{~m}_{1}+\mathrm{m}_{2}+\mathrm{m}_{3}+\mathrm{m}_{4}$ & 61 byte \\
\hline$p t 5$ & $\mathrm{t}_{5}$ & $\mathrm{t}_{1}, \mathrm{t}_{2}, \mathrm{t}_{3}, \mathrm{t}_{4}$ & $\mathrm{~m}_{1}, \mathrm{~m}_{2}, \mathrm{~m}_{3}, \mathrm{~m}_{4}, \mathrm{~m}_{5}$ & 65 byte \\
\hline
\end{tabular}

Pattern $p_{t 3}$. User has status $\beta=0$ at time $t_{1}, t_{2}$. The postponed messages are $M=\left\{m_{1}+m_{2}\right\}$. When user active at time $\mathrm{t}_{3}$. Total received messages are $M=\left\{m_{1}+m_{2}+m_{3}\right\}$.

Pattern $p_{t 4}$.User has status $\beta=0$ at time $t_{1}, t_{2}, t_{3}$. The postponed messages are $M=\left\{m_{1}+m_{2}+m_{3}\right\}$. When user active at time $\mathrm{t}_{4}$. Total received messages are $M=\left\{m_{1}+m_{2}+m_{3}+m_{4}\right\}$.

Pattern $p_{t 5}$. User has status $\beta=0$ at time $t_{1}, t_{2}, t_{3}, t_{4}$. The postponed messages are $M=\left\{m_{1}+m_{2}+m_{3}+m_{4}\right\}$. When user active at time t5. Total received messages are $M=\left\{m_{1}+m_{2}+m_{3}+m_{4}+m_{5}\right\}$.

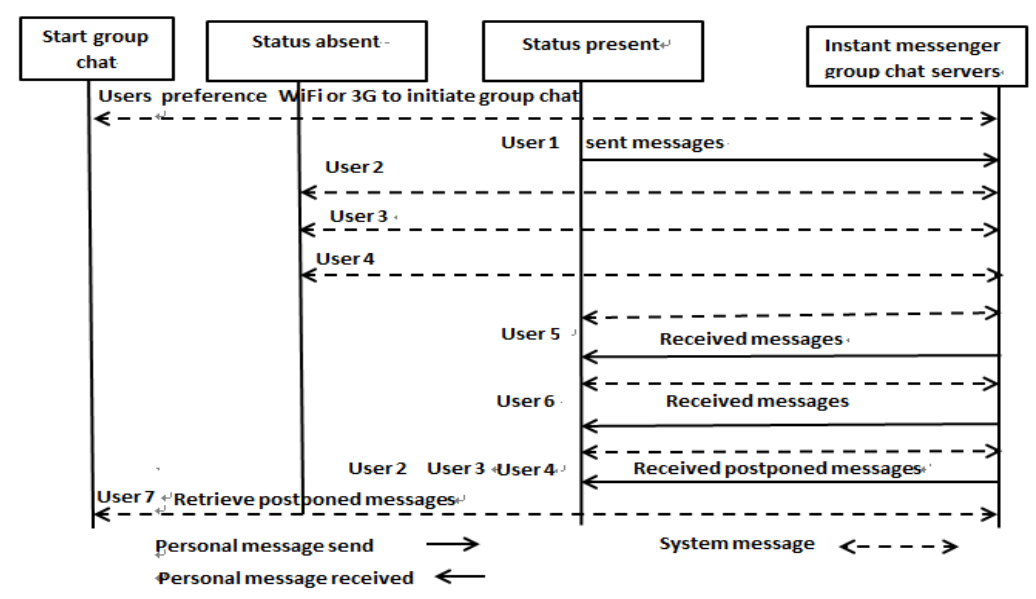

Figure 7. The Following Flow Diagram and Steps Describe Proposed Energy Efficient Group Chat Messages Sending and Receiving Scenario

\subsection{Group Chat Messages Send and Receive Work Flow}

To better understand the processes in Figure 6. We present work flow in Figure 7, by describing energy efficient group chat messages by send and receive. The energy efficient group chat flow details are described below:

1. To start group chat IM user choose from 3 options to prefer $3 \mathrm{G}$ cellular or WiFi to join the group chat. 
2. Group Chat server is responsible for enabling (user active) and disables (user inactive) sending group chat messages by identify each IM user identity in group chat.

3. User 1 has chosen option 1 prefer to switch between $3 \mathrm{G}$ cellular or WiFi network to start a group chat. User 1 is active in group chat. At this point, the user is ready to participate. The user 1 sends message to the group chat.

4. User 2,3 and 4 have chosen option 2 to prefer WiFi only. User 2,3 and 4 has inactive in group chat. This point user 2, 3 and 4 disables to receive any messages (personal message) from group chat.

5. User 5,6 have chosen option 1 prefer to switch between $3 \mathrm{G}$ cellular or WiFi to start a group chat. User 5,6 are active in group chat. At this point, the User 5 and 6 has received group chat message sent by User 1 .

6. User 2,3 and 4 have changed inactive status to active in group chat. This point user 2,3 and 4 are enabled to receive group chat message (personal message) sent by User 1 .

7. User 7 has chosen option 3 prefer to receive chat messages history from chat server.

\section{Evaluation}

We evaluate the proposed energy efficient group chat for instant messenger. We first describe the measurement tool and test environment setup. Finally, results about a cost of energy efficient group chat. We evaluate using test Android Push Notification [36] androidpn (an open source code project used for messaging). Androidpn assumed as an instant messenger chat server and used to send messages to smartphone.

\subsection{Measurement Tool}

We recorded test group chat messages energy consumption by Monsoon meter [20] and network traffic by tcpdump in Samsung 19300 Galaxy S III smartphone. Figure 8 shows a Dell desktop computer used to log power monitor traces console. Monsoon meter supplies a stable 4.2 voltage to the smartphone and tcpdump ran on a smartphone to capture network traffic. We conducted a test on $3 \mathrm{G}$ cellular internet service from CMCC (China mobile Communication Corporation) cellular network data connection and setting a WiFi access point in Dell desktop computer in our computer laboratory LAN to access WiFi internet service in Samsung I9300 Galaxy S III smartphone. To limit others application network traffic"ES Task Manager (Task Killer)" app was used to kill additional apps running in a smartphone.

\subsection{Test Environment Setup}

The five test conversion messages from Table 1 sent to smartphone by Android Push Notification (Androidpn). Androidpn acts as a group chat server to send messages to a smartphone. Figure 9 shows a test conversion messages receive by smartphone. Android Push Notification is an open source code project that pushes messages notification which supports android. It supports XMPP (Extensible Messaging and Presence Protocol) the most famous instant messenger protocol used by Gtalk, Facebook chat etc., The androidpn supports XMPP protocol which support asynchronous bidirectional messages sent and received between smartphone and server. It means not only server sends messages to the smartphone, but a smartphone can also send messages back to the server. Monsoon meter and tcpdump tool were used to metered results on a smartphone. 


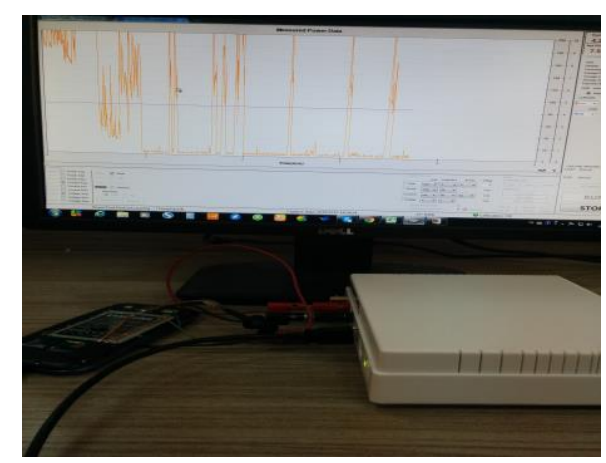

Figure 8. Experimental Setup to Measure Network Traffic and Power Measurement

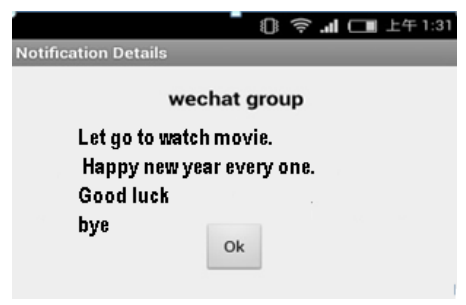

\section{Figure 9. Test Conversion Messages to Smartphone}

\subsection{Energy Saving Cost of Group Chat}

The energy cost to network preferences and user state awareness traffic pattern are as follows.

Results Table 3 shows energy consumption results of network preferences 1 to 3 . Total 5 message conversations refer to Table 1 postponed at 5 possible different traffic patterns from Table 2. On selection of user preference $1 \phi P=\left\{p_{1}\right\}=176 \mathrm{~mA}$ in pattern $1, \phi P=\left\{p_{1}, p_{2}\right\}=$ $200 \mathrm{~mA}$ in pattern $2, \phi P=\left\{p_{1}, p_{2}, p_{3}\right\}=107 \mathrm{~mA}$ in pattern $3, \phi P=\left\{p_{1}, p_{2}, p_{3}, p_{4}\right\}=111$ $\mathrm{mA}$ in pattern 4 and $\phi P=\left\{p_{1}, p_{2}, p_{3}, p_{4}, p_{5}\right\}=84.7 \mathrm{~mA}$ in pattern 5. Results in preference 2 WiFi are $\phi P=\left\{p_{1}\right\}=89 \mathrm{~mA}$ in $1, \phi P=\left\{p_{1}, p_{2}\right\}=99 \mathrm{~mA}$ in $2, \phi P=\left\{p_{1}, p_{2}, p_{3}\right\}=84 \mathrm{~mA}$ in $3, \phi P=\left\{p_{1}, p_{2}, p_{3}, p_{4}\right\}=82 \mathrm{~mA}$ in pattern 4 and $\phi P=\left\{p_{1}, p_{2}, p_{3}, p_{4}, p_{5}\right\}=77 \mathrm{~mA}$ in pattern 5. Results in preference $3 \mathrm{WiFi} \phi P=\left\{p_{1}, p_{2}, p_{3}, p_{4}, p_{5}\right\}=77 \mathrm{~mA}$ and preference $33 \mathrm{G}$ $\phi P=\left\{p_{1}, p_{2}, p_{3}, p_{4}, p_{5}\right\}=84.7$. The preference 2 to receive group chat has the lowest energy consumption. The lowest postponed messages are referred to pattern 1 is not applicable to preferences 1 and 2. Preference $3 \mathrm{WiFi}$ and 3G cellular are applicable to pattern 5 only and user can retrieve message history within 7 days expiry. Pattern 2 has the highest energy consumption compare with pattern 1, 3, 4 and 5. In pattern 5, IM user is inactive for longest time interval. The highest postponed messages are refer to pattern 1 and obviously highest energy saving from any other network preference. The results to initiate group chat by network preference traffic pattern energy saving between $13.7 \%$ to $32 \%$ for $3 \mathrm{G}$ cellular and between $21 \%$ to $27 \%$ for $\mathrm{WiFi}$, users. 
Table 3. Energy Consumption Results of Network Preferences and User Status Awareness Traffic Pattern

\begin{tabular}{|l|l|l|l|l|l|}
\hline \multicolumn{1}{|c|}{ Preferences } & Pattern 1 & Pattern 2 & Pattern 3 & Pattern 4 & \multicolumn{1}{|c|}{ Pattern 5 } \\
\hline Messages & \multicolumn{1}{|c|}{$\begin{array}{l}\mathrm{M}=\mathrm{m}_{1} \\
\mathrm{M}=\mathrm{m}_{1}+\mathrm{m}_{2}+\mathrm{m}_{2} \\
+\mathrm{m}_{3}\end{array}$} & $\begin{array}{c}\mathrm{M}=\mathrm{m}_{1}+\mathrm{m}_{2} \\
+ \\
\mathrm{m}_{3}+\mathrm{m}_{4}\end{array}$ & $\begin{array}{c}\mathrm{M}=\mathrm{m}_{1}+\mathrm{m}_{2}+\mathrm{m}_{3} \\
+\mathrm{m}_{4}+\mathrm{m}_{5}\end{array}$ \\
\hline $\begin{array}{l}\text { Preference 1 } \\
\text { 3G cellular }\end{array}$ & $176 \mathrm{~mA}$ & $200 \mathrm{~mA}$ & $107 \mathrm{~mA}$ & $111 \mathrm{~mA}$ & $84.7 \mathrm{~mA}$ \\
\hline $\begin{array}{l}\text { Preference 2 } \\
\text { WiFi }\end{array}$ & $89 \mathrm{~mA}$ & $99 \mathrm{~mA}$ & $84 \mathrm{~mA}$ & $82 \mathrm{~mA}$ & $77 \mathrm{~mA}$ \\
\hline $\begin{array}{l}\text { Preference 3 } \\
\text { Wi-Fi }\end{array}$ & N/A & N/A & N/A & N/A & $77 \mathrm{~mA}$ \\
\hline $3 G$ cellular & N/A & N/A & N/A & N/A & $84.7 \mathrm{~mA}$ \\
\hline
\end{tabular}

Based on the results above, we use the correlation relationship between energy saving cost of network preference and postponed message in user status awareness traffic pattern. Correlation is a mathematical model to recognize how variables $\mathrm{M}$ and $\varnothing \mathrm{P}$ influence each other. The variable $M$ denotes $M \|(3,26,52,61,65)$ postponed messages in traffic pattern $1 p t_{1}=m_{1} 3$ bytes, pattern $2 p t_{2}=m_{1}+m_{2} 26$ bytes, pattern $3 p t_{3}=m_{1}+m_{2}+m_{3} 52$ bytes, $p t_{4}=m_{1}+m_{2}+m_{3}+m_{4} 61$ bytes and $p t_{5}=m_{1}+m_{2}+m_{3}+m_{4}+m_{5} 65$ bytes. The variable $\varnothing \mathrm{P}$ denotes energy cost to network preferences. In $3 \mathrm{G}$ cellular $\phi P \|(176,200,107,111,84.7)$ and WiFi network $\phi P \|(89,99,84,82,77)$. The correlation defined as in equation 1.

$\rho(M, \phi P)=\frac{\operatorname{Cov}(M, \phi P)}{\sqrt{\operatorname{Var}(M) \operatorname{Var}(\phi P)}}$

$r=S_{M \phi P} / \sqrt{S_{M M} S_{\phi P \phi P}}$

The sample correlation coefficient $r$ between two samples $\mathrm{M}_{\mathrm{i}}$ and $\varnothing \mathrm{P}_{\mathrm{j}}$ is defined as in equation 2 .

To find correlation relationship between $\left(\mathrm{M}_{\mathrm{i}}\right)$ and $\left(\varnothing \mathrm{P}_{\mathrm{j}}\right)$.

First, we find correlation relationship denoted by $\mathrm{r}_{1}$ in $\left(\mathrm{M}_{\mathrm{i}}\right)$ and $\left(\varnothing \mathrm{P}_{\mathrm{i}}\right)$ in $3 \mathrm{G}$ cellular.

$M \|(3,26,52,61,65)$

$\phi P \|(176,200,107,111,84.7)$

$r=-0.86$

Second, we find correlation relationship denote $\mathrm{r}_{2}$ in $\left(M_{\boldsymbol{i}}\right)$ and $(\varnothing P \boldsymbol{i})$ in WiFi network

$M \|(3,26,52,61,65)$

$\phi P \|(89,99,84,82,77)$

$r=-0.77$ 
Correlation analysis results in $\mathrm{r} 1=-0.86$ and $\mathrm{r} 2=-0.77$ both have a negative relationship between energy saving cost of network preference and postponed message in user status awareness traffic pattern. The r1 represent a relationship between energy saving cost of network preference and postponed message in $3 \mathrm{G}$ cellular and $\mathrm{r} 2$ represent a relationship between energy saving cost of network preference and postponed message in WiFi network. In summary, as an increase in traffic pattern postponed messages decrease in energy cost to network preferences in a smartphone. Hence, network preferences and postponed messages dependent on user state awareness traffic pattern in a group to save a lot of joules.

\section{Conclusion and Future Work}

When the traffic is direct without user interaction running in background traffic in $3 \mathrm{G}$ cellular or WiFi network can result in energy wastage in a smartphone. We analyse group chat traffic pattern and network data usage collected from 36 IM QQ and WeChat users. It reveals current IM WeChat and QQ apps IM users cannot set network preferences such as $3 \mathrm{G}$ and WiFi network and traffic pattern is not based on user status awareness in group chat. We find IM app group chat for a large volume of group chat messages in background traffic overheads on smartphone energy consumption. Recently, many researchers proposed bundled, batched and aggregate data transmission techniques to overcome undisciplined data transmission energy wastage. Our proposed IM network preference ( $3 \mathrm{G}$ cellular or WiFi) and traffic pattern dependent on user status awareness in IM group chat, metering this technique to proposed energy efficient group chat energy consumption results show significant improvements in saving energy.

Future work can be extended to studying multimedia message sharing, which appears interesting in energy consumption prospective. Multimedia messages (images, web links, video and audio) consume much more energy compare to text messages due to their data size. Sharing multimedia messages to buddy list recipients is dependent on action of the IM app user. Studying the IM app user sharing behavior can reveal impact on energy consumption on smartphone.

\section{Acknowledgment}

This work is supported by the National Natural Science Foundation of China under Grant No. 61309025, 61402543 and 61472451, Hunan Provincial Natural Science Foundation of China under Grant No. 13JJ4016 and International Science and Technology Cooperation Programme of China under Grant No. 2013DFB10070.

\section{References}

[1] A. Pathak, A. Jindal, Y. C. Hu, and S. P. Midkiff, "What is keeping my phone awake?: characterizing and detecting no-sleep energy bugs in smartphone apps," in Proceedings of the 10th international conference on Mobile systems, applications, and services, pp. 267-280, ACM, (2012).

[2] A. Parate, M. Böhmer, D. Chu, D. Ganesan, and B. M. Marlin, "Practical prediction and prefetch for faster access to applications on mobile phones," in Proceedings of the 2013 ACM international joint conference on Pervasive and ubiquitous computing, pp. 275-284, ACM, (2013).

[3] S. K. Baghel, K. Keshav, and V. R. Manepalli, "An investigation into traffic analysis for diverse data applications on smartphones," in Communications (NCC), 2012 National Conference on, pp. 1-5, IEEE, (2012).

[4] G. Maier, F. Schneider, and A. Feldmann, "A first look at mobile hand-held device traffic," in Passive and Active Measurement, pp. 161-170, Springer, (2010).

[5] F. Xu, Y. Liu, T. Moscibroda, R. Chandra, L. Jin, Y. Zhang, and Q. Li, "Optimizing background email sync on smartphones," in Proceeding of the 11th annual international conference on Mobile systems, applications, and services, pp. 55-68, ACM, (2013).

[6] E. J. V. Alonso, S. Andersson, and S. Nadjm-Tehrani, "When mice consume like elephants: Instant messaging applications,"

[7] S. Millward, "https://www.techinasia.com/wechat-438-million-active-users-q2-2014/," 10 (2014). 
[8] S. Deng and H. Balakrishnan, "Traffic-aware techniques to reduce $3 \mathrm{~g} /$ lte wireless energy consumption," in Proceedings of the 8th international conference on Emerging networking experiments and technologies, pp. 181-192, ACM, (2012).

[9] A. Misra and L. Lim, "Optimizing sensor data acquisition for energy-efficient smartphone-based continuous event processing," in Mobile Data Management (MDM), 2011 12th IEEE International Conference on, vol. 1, pp. 88-97, IEEE, (2011).

[10] G. M. Weiss and J. W. Lockhart, "The impact of personalization on smartphone-based activity recognition," in AAAI Workshop on Activity Context Representation: Techniques and Languages, (2012).

[11] J. W. Lockhart, T. Pulickal, and G. M. Weiss, "Applications of mobile activity recognition," in Proceedings of the 2012 ACM Conference on Ubiquitous Computing, pp. 1054-1058, ACM, (2012).

[12] T. O. Oshin, S. Poslad, and A. Ma, "Improving the energy-efficiency of gps based location sensing smartphone applications," in Trust, Security and Privacy in Computing and Communications (TrustCom), 2012 IEEE 11th International Conference on, pp. 1698-1705, IEEE, (2012).

[13] L. Zhang, J. Liu, H. Jiang, and Y. Guan, "Senstrack: energy-efficient location tracking with smartphone sensors," (2013).

[14] PowerTutor, "http://powertutor.org/.," 03 (2011).

[15] C. Yoon, D. Kim, W. Jung, C. Kang, and H. Cha, "Appscope: Application energy metering framework for android smartphone using kernel activity monitoring," in USENIX ATC, (2012).

[16] A. Carroll and G. Heiser, "An analysis of power consumption in a smartphone," in Proceedings of the 2010 USENIX conference on USENIX annual technical conference, pp. 21-21, (2010).

[17] F. Ding, F. Xia, W. Zhang, X. Zhao, and C. Ma, "Monitoring energy consumption of smartphones," in Internet of Things (iThings/CPSCom), 2011 International Conference on and 4th International Conference on Cyber, Physical and Social Computing, pp. 610-613, IEEE, (2011).

[18] C. Thompson, D. C. Schmidt, H. A. Turner, and J. White, "Analyzing mobile application software power consumption via model-driven engineering.," in PECCS, pp. 101-113, (2011).

[19] Intel, "http://software.intel.com/en-us/articles/intel-power-gadget-20," 08 (2014).

[20] I. Monsoon Solutions, "www.msoon.com/labequipment/powermonitor/," 03 (2014).

[21] F. Qian, Z. Wang, A. Gerber, Z. Mao, S. Sen, and O. Spatscheck, "Profiling resource usage for mobile applications: a cross-layer approach," in Proceedings of the 9th international conference on Mobile systems, applications, and services, pp. 321-334, ACM, (2011).

[22] O. Shoukry, M. Abd El-Mohsen, J. Tadrous, H. El Gamal, T. ElBatt, N. Wanas, Y. Elnakieb, and M. Khairy, "Proactive scheduling for content pre-fetching in mobile networks," in Communications (ICC), 2014 IEEE International Conference on, pp. 2848-2854, IEEE, (2014).

[23] S. Chen, Z. Yuan, and G.-M. Muntean, "An energy-aware multipath-tcp-based content delivery scheme in heterogeneous wireless networks," in Wireless Communications and Networking Conference (WCNC), 2013 IEEE, pp. 1291-1296, IEEE, (2013).

[24] R. Friedman, A. Kogan, and Y. Krivolapov, "On power and throughput tradeoffs of wifi and bluetooth in smartphones," Mobile Computing, IEEE Transactions on, vol. 12, no. 7, pp. 1363-1376, (2013).

[25] G. Kalic, I. Bojic, and M. Kusek, "Energy consumption in android phones when using wireless communication technologies," in MIPRO, 2012 Proceedings of the 35th International Convention, pp. 754-759, IEEE, (2012).

[26] C. Paasch, G. Detal, F. Duchene, C. Raiciu, and O. Bonaventure, "Exploring mobile/wifi handover with multipath tcp," in Proceedings of the 2012 ACM SIGCOMM workshop on Cellular networks: operations, challenges, and future design, pp. 31-36, ACM, (2012).

[27] D. Wischik, C. Raiciu, A. Greenhalgh, and M. Handley, "Design, implementation and evaluation of congestion control for multipath tcp," in Usenix NSDI, (2011).

[28] A. Balasubramanian, R. Mahajan, and A. Venkataramani, "Augmenting mobile 3g using wifi," in Proceedings of the 8th international conference on Mobile systems, applications, and services, ACM, (2010).

[29] N. Balasubramanian, A. Balasubramanian, and A. Venkataramani, "Energy consumption in mobile phones: a measurement study and implications for network applications," in Proceedings of the 9th ACM SIGCOMM conference on Internet measurement conference, pp. 280-293, ACM, (2009).

[30] J. Huang, F. Qian, A. Gerber, Z. M. Mao, S. Sen, and O. Spatscheck, "A close examination of performance and power characteristics of $4 \mathrm{~g}$ lte networks," in Proceedings of the 10th international conference on Mobile systems, applications, and services, pp. 225-238, ACM, (2012).

[31] $\mathrm{H} . \mathrm{Li}$ and $\mathrm{S} . \mathrm{Xu}$, "Challenges and strategies of higher education against the background of mobile media," in International Conference on Education, Language, Art and Intercultural Communication (ICELAIC-14), Atlantis Press, (2014).

[32] A. R. M. Basher and B. C. Fung, "Analyzing topics and authors in chat logs for crime investigation," Knowledge and information systems, vol. 39, no. 2, pp. 351-381, (2014).

[33] A. Orebaugh, J. Kinser, and J. Allnutt, "Visualizing instant messaging author writeprints for forensic analysis," in Proceedings of the Conference on Digital Forensics, Security and Law, pp. 191-214, (2014). 
[34] L. Zhang, C. Xu, P. H. Pathak, and P. Mohapatra, "Characterizing instant messaging apps on smartphones," in Passive and Active Measurement, pp. 83-95, Springer, (2015).

[35] E. J. Vergara Alonso, "Exploiting energy awareness in mobile communication," (2013).

[36] Androidpn, "http://sourceforge.net/projects/androidpn/?source=directory," 03 (2014).

\section{Authors}

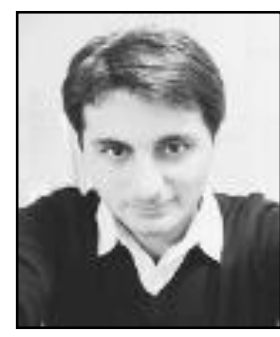

Pinial Khan Butt received master's degree in Telecommunication Technology from University of Sindh, Pakistan in 2002. He is currently a Doctoral Degree Candidate at the School of Information Science and Engineering, Central South University, Changsha, Hunan China. His research interests include E-Commerce, Telecommunication Technology and Energy Efficient Mobile Computing.

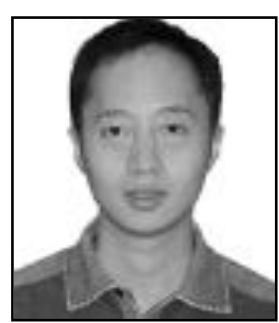

Wang Yang received B.Sc. in Computer Science and technology from National University of Defense Technology, China in 2004, and Ph.D. in Computer Science and technology from Tsinghua University, China in 2011. He is now an Assistant Professor in School of Information Science and Engineering, Central South University, China. His research interests include mobile computing, sustainable computing and information centric networking.

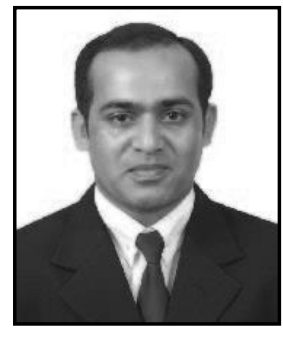

Md Zakirul Alam Bhuiyan received the $\mathrm{PhD}$ degree and the M.Eng degree from Central South University, China, in 2009 and 2013 respectively, and the BSc degree from International Islamic University Chittagong, Bangladesh, in 2005, all in Computer Science and Technology. He is currently an assistant professor (research) in the Department of Computer and Information Sciences at Temple University. $\mathrm{He}$ is a member of the Center for Networked Computing (CNC). Earlier, he worked as a post-doctoral fellow at the Central South University, China, a research assistant at the Hong Kong PolyU, and a software engineer in industries. His research focuses on dependable cyber physical systems, wireless sensor network applications, network security, and sensor-cloud computing. $\mathrm{He}$ has served as a managing guest editor, workshop chair, publicity chair, TPC member, and reviewer of international journals/conferences. $\mathrm{He}$ is a member of IEEE and a member of ACM.

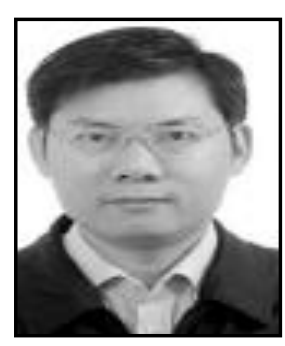

Guojun Wang received his B.Sc. in Geophysics, M.Sc. in Computer Science, and Ph.D in Computer Science from Central South University, China. He is currently a Professor of Guangzhou University and Central South University. He has been an Adjunct Professor at Temple University, USA; a Visiting Scholar at Florida Atlantic University, USA; a Visiting Researcher at the University of Aizu, Japan; and a Research Fellow at the Hong Kong Polytechnic University. His research interests include network and information security, Internet of things, and cloud computing. $\mathrm{He}$ is a distinguished member of CCF, and a member of IEEE, ACM, and IEICE. 